\title{
Bose-Einstein condensation in multilayers
}

\author{
P. Salas ${ }^{1,2}$, M. Fortes ${ }^{2}$, M. de Llano ${ }^{3}$, F.J. Sevilla ${ }^{2}$ and M.A. Solís ${ }^{2}$ \\ ${ }^{1}$ Posgrado en Ciencias e Ingeniería de Materiales, UNAM, México, D.F., MEXICO \\ ${ }^{2}$ Instituto de Fúsica, UNAM, México, D.F., MEXICO \\ ${ }^{3}$ Instituto de Investigaciones en Materiales, UNAM, México, D.F., MEXICO
}

\begin{abstract}
The critical BEC temperature $T_{c}$ of a non interacting boson gas in a layered structure like those of cuprate superconductors is shown to have a minimum $T_{c, m}$, at a characteristic separation between planes $a_{m}$. It is shown that for $a<a_{m}, T_{c}$ increases monotonically back up to the ideal Bose gas $T_{0}$ suggesting that a reduction in the separation between planes, as happens when one increases the pressure in a cuprate, leads to an increase in the critical temperature. For finite plane separation and penetrability the specific heat as a function of temperature shows two novel crests connected by a ridge in addition to the well-known BEC peak at $T_{c}$ associated with the $3 \mathrm{D}$ behavior of the gas. For completely impenetrable planes the model reduces to many disconnected infinite slabs for which just one hump survives becoming a peak only when the slab widths are infinite.
\end{abstract}

PACS numbers: 03.75.Hh;05.30.Jp;67.25.bh;74.78.Fk

Since London first suggested $\underline{1}$ that superfluidity in liquid ${ }^{4} \mathrm{He}$ might well be a manifestation of Bose-Einstein condensation (BEC) of the helium atoms before interatomic interactions are "switched on," BEC in layered systems began to be studied to understand helium films $2,3,4$. The discovery of high- $T_{c}$ superconductivity stimulated renewed interest in compounds with layered structures ${ }^{5}$ in which a BEC mechanism seems to be an essential feature to explain high critical temperatures ${ }^{6}$. The so-called "Uemura plot" $\underline{\underline{z}}$ of data from muon-spin relaxation $(\mu \mathrm{SR})$, neutron and Raman scattering, and angle-resolved photoemission (ARPES) measurements exhibits $T_{c}$ vs Fermi temperatures $T_{F} \equiv E_{F} / k_{B}$ where $E_{F}$ the Fermi energy and $k_{B}$ the Boltzmann constant. Empirical $T_{c}$ s of many cuprates straddle a line parallel to the Uemura-plot diagonal line associated with the simple BEC formula $T_{0} \simeq 3.31 \hbar^{2} n_{B}^{2 / 3} / m k_{B} \simeq 0.218 T_{F}$ corresponding to an ideal gas of bosons of mass $m=2 m^{*}$ and number density $n_{B}=n_{s} / 2$ where $m^{*}$ is the individualcharge-carrier effective mass and $n_{s}$ their number density. The parallel line of data is shifted down from $T_{0}$ by a factor of 4-5. This has been judged $\frac{8}{-}$ a "fundamental importance of the BEC concept in cuprates." In addition, the possibility of creating BECs or superfluidity of ultracold fermions ${ }^{9}$ in optical lattices 10 , along with the expected observation of BEC of excitons (electron-hole pairs) in semiconductors 11 , have further revived theoretical and experimental ${ }^{12,13}$ efforts to better understand the behavior of quantum gases in layered geometries.

Most models based on layered structures ${ }^{6,15}$ simulating quasi-2D high- $T_{c}$ superconductors, or other to study BEC $16,17,18$, rely on a single-boson hopping interaction term producing nearest-interlayer couplings in one spatial dimension while moving freely in the other two directions. The energy spectrum is typically of the form $\epsilon_{\mathbf{k}}=$ $\hbar^{2}\left(k_{x}^{2}+k_{y}^{2}\right) / 2 m+\epsilon_{k_{z}}$ with $\epsilon_{k_{z}}=\left(\hbar^{2} / M a^{2}\right)\left(1-\cos k_{z} a\right)$ where $a$ is the plane separation and the constant $\hbar^{2} / M a^{2}$ is a measure of the bosonic Cooper pair hopping probability between planes. In the case of $\mathrm{CuO}_{2}$ planes in cuprate superconductors a boson effective mass $M$ in the $z$-direction is used with $m$ the mass in the $x$-, $y$-directions while $M>m$ reflects cuprate anisotropy. For $k_{z} a \ll 1$ one recovers the expected result $\epsilon_{k_{z}}=\hbar^{2} k_{z}^{2} / 2 M$.

Motivated by possible applications to quasi-2D superconductors or to ${ }^{4} \mathrm{He}$ films, in this Brief Report we discuss critical temperature $T_{c}$ and the specific heat $C_{V}$ results in a non-interacting boson gas of $N$ particles within an infinite multilayered structure of equally-spaced planes of variable penetrability stacked in the $z$-direction.

The system of parallel planes is simulated with an external periodic delta potential of the 1D Kronig-Penney (KP) type along the $z$-direction while bosons are free in the two remaining directions. Two important differences with previous studies $6,16,17,18$ are: a) the bosons are allowed to move over all space through permeable planes instead of constraining them to move only over the plane surfaces, and b) the anisotropy expected from a layered material is introduced naturally by the delta KP potential. In addition, the delta strengths avoid the artificial introduction of large masses and hopping parameters in the $z$-direction to tune the mobility or tunneling across planes. At very low energies the KP potential reduces to the energy expression $\epsilon_{k_{z}}$ used in Refs $, 6,16,17,18$ which only takes into account the lowest allowed energy band. If $T_{0}$ is the critical BEC temperature of the free $3 \mathrm{D}$ boson gas and the associated thermal wavelength is $\lambda_{0} \equiv h / \sqrt{2 \pi m k_{B} T_{0}}$ a dimensionless "impenetrability" $P_{0} \equiv P \lambda_{0} \geqslant 0$ of layers is introduced in terms of the KP penetrability parameter $P$ [see (10) below]. Thus, $P_{0}=0$ implies perfect layer transparency and $P_{0}=\infty$ fully opaque layers. In the latter limit, our model reduces to that of an infinite number of uncoupled slabs of thickness $a$ and infinite lateral extent. The specific heat has been obtained in these systems to model ${ }^{4} \mathrm{He}$ thinfilm properties $19,20,22,23$ and finite-size effects on BEC 24 . In these infinite slabs the BEC critical temperature is zero but the specific heat shows a smooth maximum at a temperature that depends on the slab thickness. Some authors $22,23,24$ have associated this "hump" with a BEC signature. However, we show here that for finite $P_{0}$ this 
hump coexists with a nonzero critical BEC temperature in addition to a second maximum and when $P_{0} \rightarrow \infty$ the BEC temperature goes to zero whereas the hump persists to become the familiar BEC with a cusped sharp peak only in the limit $a / \lambda_{0} \rightarrow \infty$.

The Schrödinger equation for a boson of mass $m$ is separable in the $x, y$ and $z$-directions so that the single-particle energy as a function of the wavevector $\mathbf{k}=\left(k_{x}, k_{y}, k_{z}\right)$ is simply $\varepsilon_{\mathbf{k}}=\varepsilon_{k_{x}}+\varepsilon_{k_{y}}+\varepsilon_{k_{z}}$, where $\varepsilon_{k_{x}, k_{y}}=\hbar^{2} k_{x, y}^{2} / 2 m$ with $k_{x, y}=2 \pi n_{x, y} / L$ and $n_{x, y}=$ $0, \pm 1, \pm 2, \cdots$, i.e., in the $x$ and $y$-directions particles are free and we assume periodic boundary conditions in a box of size $L$. In the $z$-direction the particles are subject to the KP periodic delta (or "Dirac comb") potential in $1 \mathrm{D}^{21}$ and the energies $\varepsilon_{k_{z}}$ are implicit in the transcendental equation

$$
P a \frac{\sin \alpha a}{\alpha a}+\cos \alpha a=\cos k_{z} a
$$

where $\alpha^{2} \equiv 2 m \varepsilon_{k_{z}} / \hbar^{2}$ and $\hbar^{2} P / m$ is the delta-interaction strength or equivalently, the layer impenetrability parameter. The energy $\varepsilon_{k_{z}, j}$ depends on the parameters $P$ and $a$ where the band structure is labeled with the index $j=1,2, \ldots$ The trivial free-particle energy dispersion relation in the $z$-direction, $\varepsilon_{k_{z}, j} \rightarrow \hbar^{2} k_{z}^{2} / 2 m$, is recovered in the limit $P \rightarrow 0$, while $P \rightarrow \infty$ yields $\sin (\alpha a) \rightarrow 0$ which corresponds to a system of confined bosons inside a semi-infinite slab of width $a$, a situation extensively discussed in the literature ( $\sec ^{23}$ and references therein). For $\alpha a \ll 1$, (11) can be expanded as

$$
\varepsilon_{k_{z}} \simeq \frac{\hbar^{2}}{2 m a^{2}} \frac{P a+\left(1-\cos k_{z} a\right)}{1 / 2+P a / 6} \underset{P a \ll 1}{\longrightarrow} \frac{\hbar^{2}}{m a^{2}}\left(1-\cos k_{z} a\right)
$$

where the last expression is the dispersion relation used in ${ }^{6,16,18}$. For finite $P$, bosons tunnel through the layers as a more realistic model would demand.

The grand potential $\Omega(T, V, \mu)=-p V$ (with $p$ the pressure, $V$ the volume and $\mu$ the chemical potential) leads to all the system thermodynamic properties. In our multilayered system it becomes, after integrating over $k_{x}$ and $k_{y}$,

$\Omega(T, V, \mu)=\Omega_{0}-\frac{1}{\beta^{2}} \frac{V m}{(2 \pi)^{2} \hbar^{2}} \sum_{j=1}^{\infty} \int_{-\pi / a}^{\pi / a} d k_{z} g_{2}\left(z e^{-\beta \varepsilon_{k_{z} j}}\right)$

where $z \equiv e^{\beta \mu}$ is the fugacity, $\beta \equiv 1 / k_{B} T$, and we have explicitly separated the contribution $\Omega_{0} \equiv k_{B} T \ln [1-$ $\left.z e^{-\beta \varepsilon_{0}}\right]$ of the lowest-energy state $\varepsilon_{0}$. Here $g_{\sigma}(t)$ stands for the Bose function ${ }^{25}$. The integration is over the first Brillouin zone and the sum is over the allowed bands. Note that the ground-state energy $\varepsilon_{0}$ depends on both $P$ and $a$ in (11).

The average number of particles $N=-(\partial \Omega / \partial \mu)_{T, V}$ for a free boson gas in the multilayered configuration is then

$$
N=N_{0}(T)-\frac{V m}{(2 \pi)^{2} \hbar^{2}} \frac{1}{\beta} \sum_{j=1}^{\infty} \int_{-\pi / a}^{\pi / a} d k_{z} \ln \left[1-z e^{-\beta \varepsilon_{k_{z} j}}\right]
$$

where $N_{0}(T) \equiv\left[z^{-1} e^{\beta \varepsilon_{0}}-1\right]^{-1}$ is the number of particles condensed in the ground state $\varepsilon_{0}$ while the last term in (3) is the number of particles in excited states. As usual, the BEC critical temperature $T_{c}$ is obtained by finding the temperature below which the fractional number of particles in the ground state just ceases to be negligible upon cooling and the chemical potential reaches its maximum value $\varepsilon_{0}>0$ making the fugacity equal $z_{0}=e^{\beta_{c} \varepsilon_{0}}$. For convenience and clarity we use the same particle density $N / V$ in the layered system as that for the free ideal Bose gas (IBG) with the BEC critical temperature $T_{0}$. Thus $k_{B} T_{0}$ serves as an energy scale while $\lambda_{0} \equiv h / \sqrt{2 \pi m k_{B} T_{0}}$ provides a length scale.

The effects of the KP layers on BEC are shown in Fig. 1 where $T_{c} / T_{0}$ decreases as $P_{0}$ increases for different values of $a$. Note that the critical temperature is very sensitive to the number of energy bands intervening in the numerical calculations. Here, it is sufficient to include 10 bands to achieve convergence for the values of $a$ and $P_{0}$ shown in the figure. When $P_{0}=0$ (perfect barrier transparency) we recover the IBG results. In the opposite limit $P_{0} \rightarrow \infty$ (impenetrable barriers) $T_{c} / T_{0}$ vanishes although strictly speaking the model does not become a true two-dimensional system.

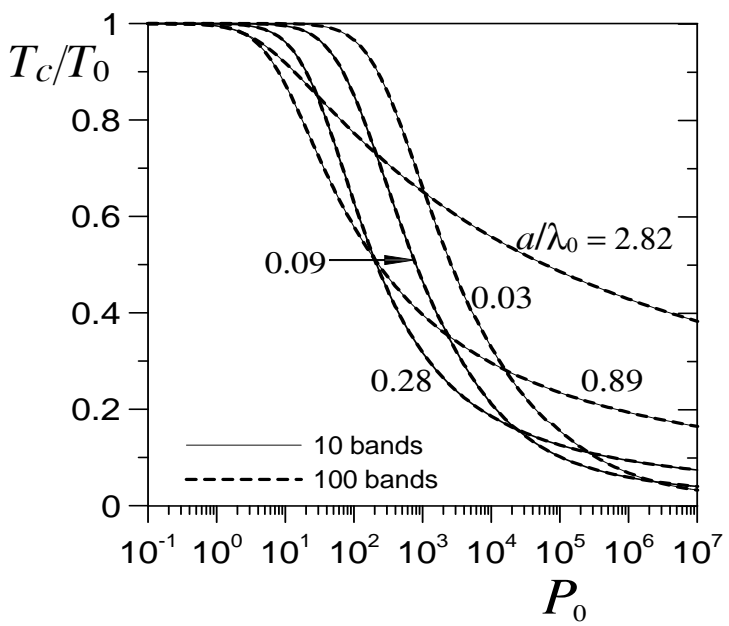

FIG. 1: Critical temperature $T_{c}$ in units of $T_{0}$ as a function of $P_{0}$ for different values of $a / \lambda_{0}$.

A novel result shown in Fig. 2 is that for finite $P_{0}$, $T_{c} / T_{0}$ diminishes from 1 down to a minimum value as the plane separation $a$ decreases. For $P_{0}$ spanning at least five orders of magnitude (see Table \) the criticaltemperature minima show a linear dependence with $a_{m}$ so that $T_{c, m}=\left(T_{0} / \lambda_{0}\right) a_{m}$ or $\left(\lambda_{0} / \lambda_{c, m}\right)^{2}=a_{m} / \lambda_{0}$, where the subscript $m$ means minimum and $\lambda_{c, m}$ is the thermal wave length associated to $T_{c, m}$. Since $T_{c, m} \rightarrow 0$ and 
TABLE I: Values of plane separation $a_{m}$ for which the critical temperature reaches its minimum for different $P_{0}$.

\begin{tabular}{cccccc}
\hline \hline$P_{0}$ & 10 & $10^{2}$ & $10^{3}$ & $10^{4}$ & $10^{5}$ \\
\hline$a_{m} / \lambda_{0}$ & 0.874 & 0.550 & 0.314 & 0.177 & 0.100 \\
$T_{c m} / T_{0}$ & 0.927 & 0.572 & 0.337 & 0.191 & 0.107 \\
\hline \hline
\end{tabular}

$a_{m} \rightarrow 0$ for $P_{0} \rightarrow \infty$ this linear relation seems to hold for all $P_{0} \gtrsim 10$. For $P_{0} \lesssim 10, T_{c, m} / T_{0}$ approaches 1 while $a_{m} \rightarrow \infty$. Surprisingly, further reduction in $a$ brings an increase in $T_{c} / T_{0}$ which asymptotically reaches unity. This can be understood from the KP dispersion relation (11) since for small values of $a \sin (\alpha a) / \alpha a \simeq 1$ and $\varepsilon_{k_{z}, j} \simeq$ $\hbar^{2} k_{z}^{2} / 2 m$ so that one recovers the 3D IBG regime when $a \rightarrow 0$, as expected. We also note in Fig. 2 that $T_{c} / T_{0}$ is not symmetric wrt the minimum value. For $a / \lambda_{0} \ll 1$, $T_{c} / T_{0}$ increases slower than for $a / \lambda_{0} \gg 1$ which reflects the plane influence on the bosons. We also note that for $a / \lambda_{0} \gg 1$ one must include more than ten energy bands to achieve convergence in the numerical calculations.

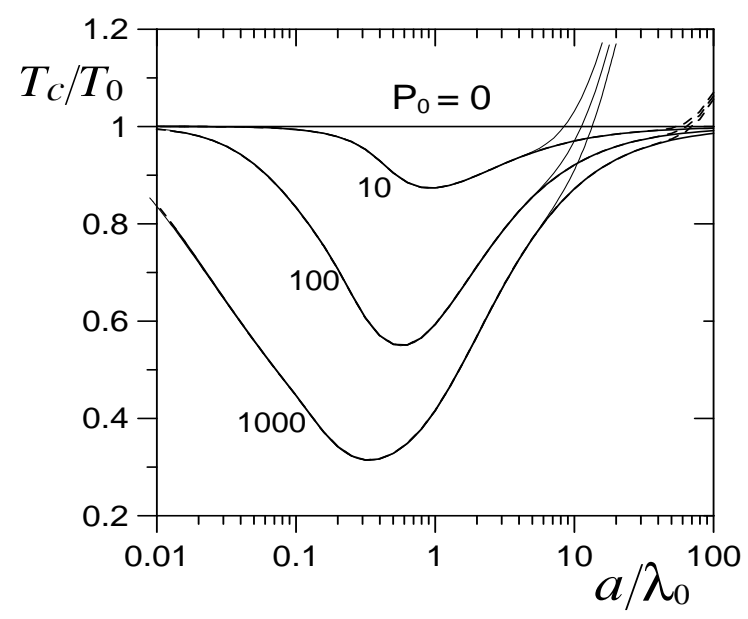

FIG. 2: Critical temperature as a function of $a / \lambda_{0}$ for different values of $P_{0}$. For $a \gg \lambda_{0}$ the critical temperature is very sensitive to the number of energy bands used in the numerical calculations. Thin, dashed and normal lines correspond to 10, 100 and 1000 bands, respectively. To attain convergence we need to consider more than ten bands (thin line) which contrasts with the behavior in the $a<\lambda_{0}$ where the inclusion of few bands is enough to obtain convergence

The specific heat $C_{V}=-T\left[\partial^{2} \Omega / \partial T^{2}\right]_{V, \mu}$ is

$$
\begin{aligned}
& \frac{C_{V}}{N k_{B}}=\frac{V m}{N(2 \pi)^{2} \hbar^{2}} \sum_{j=1}^{\infty} \int_{-\pi / a}^{\pi / a} d k_{z}\left[2 k_{B} T g_{2}\left(z e^{-\beta \varepsilon_{k_{z} j}}\right)\right. \\
& -\ln \left(1-z e^{-\beta \varepsilon_{k_{z} j}}\right)\left[2 \varepsilon_{k_{z} j}-\mu-\varepsilon_{0}+T \frac{\partial \mu}{\partial T}\right] \\
& \left.+\frac{1}{k_{B} T} \frac{\left(\varepsilon_{k_{z} j}-\varepsilon_{0}\right)\left[\varepsilon_{k_{z} j}-\mu+T \frac{\partial \mu}{\partial T}\right]}{z^{-1} e^{\beta \varepsilon_{k_{z} j}}-1}\right]
\end{aligned}
$$

where

$$
T(d \mu / d T)=\frac{-\frac{N}{V} \frac{(2 \pi)^{2} \hbar^{2}}{m}-\sum_{j=1}^{\infty} \int_{-\pi / a}^{\pi / a} d k_{z} \frac{\varepsilon_{k_{z} j}-\mu}{z^{-1} e^{\beta \varepsilon_{z z} j}-1}}{\sum_{j=1}^{\infty} \int_{-\pi / a}^{\pi / a} d k_{z}\left[z^{-1} e^{\beta \varepsilon_{k_{z} j}}-1\right]^{-1}}
$$

For $T<T_{c}$ the chemical potential $\mu=\varepsilon_{0}$ is a con-

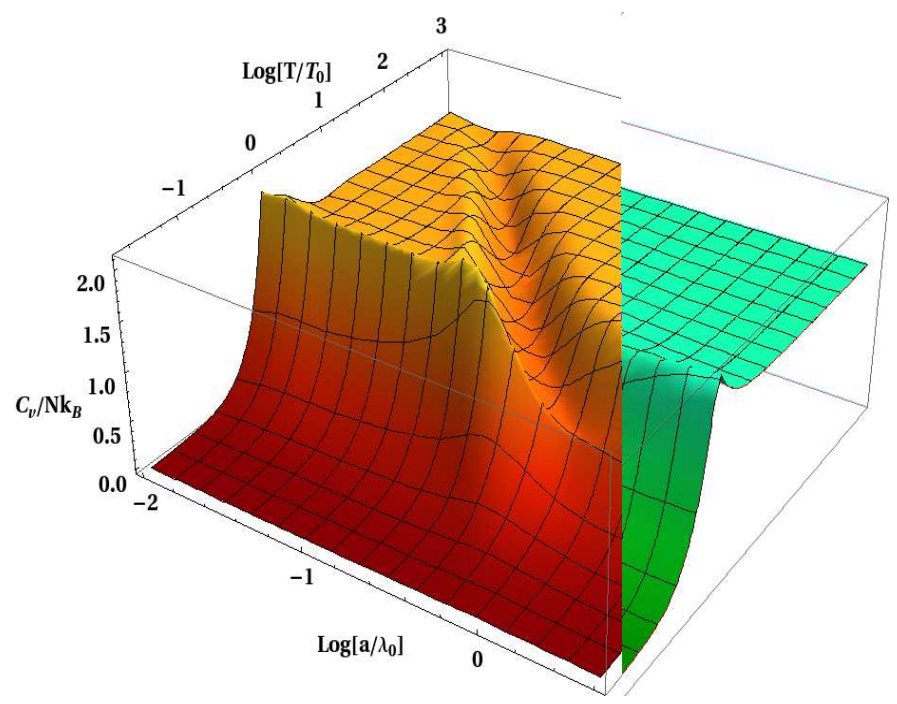

FIG. 3: 3D plot of the specific heat as function of temperature and plane separation $a$ for $P_{0}=100$.

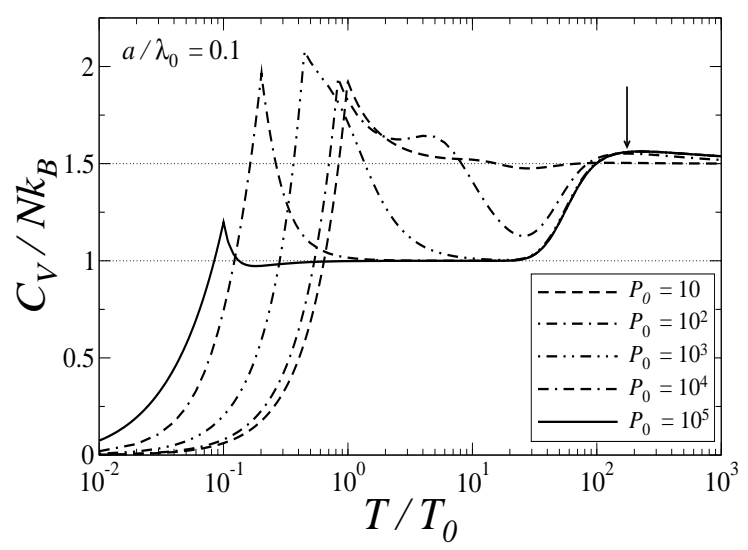

FIG. 4: Specific heat per particle as a function of temperature for several $P_{0}$ and $a / \lambda_{0}=0.1$. The two-dimensional character of the layered structure is easily seen as $P_{0}$ increases. The arrow indicates the initiation of the IBG regime

stant so that $\partial \mu / \partial T=0$ which simplifies the equation for $C_{V} / N k_{B}$.

Numerical results are summarized in Fig. 3 of $C_{V} / N k_{B}$ vs. $\log T / T_{0}$ and vs. $\log a / \lambda_{0}$ for an intermediate dimensionless penetrability $P_{0}=100$. In contrast 
with the IBG case, in addition to the familiar BEC peak for finite $P_{0}$ two humps appear that are connected by a ridge when $T>T_{c}$; ridge heights depend on the plane separation. When $a>a_{m}$ only one hump survives and becomes the BEC cusped peak in the limit $a \rightarrow \infty$ independently of $P_{0}$. Our model shows the distinct nature of the ever present BEC critical temperature plus a second characteristic temperature where $C_{V} / N k_{B}$ is maximum. In previous models (here equivalent to $\left.P_{0} \rightarrow \infty\right)^{23} \underline{24}$ this latter temperature is identified as a signature of the $\mathrm{BEC}$ critical temperature. However, our results indicate that this characteristic temperature should be associated with a different feature, namely an accumulation of bosons 26 in the lowest energy levels.

While $C_{V} / N k_{B}$ of the IBG decreases monotonically for $T>T_{c} \equiv T_{0}$, in the present model there is a region defined by $a \lesssim a_{m}$ where $C_{V} / N k_{B}$ varies nonmonotonically with $\widetilde{T}$ having a set of local minima (the ridge in Fig. 3) where $\lambda \simeq 2 a$. In this case, the singleparticle (thermal) average wavefunction nodes coincide with the plane locations resulting in the particle motion freezing out in the $z$-direction. In particular, for $a=a_{m}$ the minimum of $C_{V}(T) / N k_{B}$ attains its lowest value $\simeq 1$ so that the "ridge" reveals a closer resemblance to a $2 \mathrm{D}$ system. As $P_{0}$ increases this $2 \mathrm{D}$-like feature dominates as the ridge levels out over a broader temperature region (see Fig. 4 for $a / \lambda_{0}=0.1$ ). In addition, for temperatures below $T_{c}$ the value $\lambda \simeq 2 a$ marks a functional crossoever of specific heat from the standard 3D IBG $C_{V} \propto T^{3 / 2}$ to the $2 \mathrm{D}$ linear $C_{V} \propto T$ behavior. For sufficiently high temperatures, layer effects are negligible regardless of separa- tion $a$ or of penetrability $P_{0}$. In this regime $C_{V}(T) / N k_{B}$ approaches the IBG value. Since $C_{V}$ attains this value at the same $T$ (implying the same $\lambda$ ) we can identify an $a$-dependent correlation length that manifests itself when

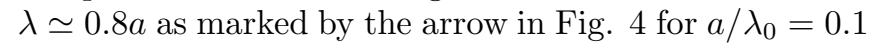
and $T / T_{0} \simeq 170 K$.

To conclude, we have shown that the BEC-like critical temperature as well as the specific heat of a boson gas between penetrable and periodically-spaced planes change drastically compared to previous models based on hopping, Hubbard $\underline{\underline{6}}$ or $\operatorname{slab}^{\underline{23}}$ configurations, or when there is no confining geometrical structure whatsoever. Even though analytical approximations for the boson dispersion relation at low energies commonly used to describe layered systems have proved to be a useful guide to give more accurate results, they are significantly improved when the more accurate dispersion relation including more than one energy band is used. The appearance of two characteristic temperatures associated with the maxima of $C_{V}(T)$ in addition to the BEC transition temperature due to the confinement parameters may explain the variation of $T_{c}$ with pressure in high- $T_{c}$ superconductors the transition is related with a BEC-like phenomenon. In the limit $P_{0} \rightarrow \infty$ (opaque or perfectly-decoupled infinite slabs of width $a$ ) the BEC-like transition temperature vanishes leaving only one maximum which becomes the usual cusped-peak BEC transition only when $a \gg \lambda_{0}$.

We acknowledge partial support from grants PAPIIT IN114708 and IN106908. F.J.S. acknowledges partial support from Conacyt-SNI-I-89774. We thank O.A. Rodríguez for assistance in preparing Fig. 3 .
1 F. London, Nature 141, 643 (1938) and Phys. Rev. 54, 947 (1938).

2 D.F. Brewer and K. Mendelssohn, Phil. Mag. 44, 340 (1953).

3 S. Mehta and F.M. Gasparini, Phys. Rev. Lett. 78, 2596 (1997).

4 Y. Kinosita et al., J. Low Temp. Phys. (2009) online 14 October; T. Matsushita, R. Toda, M. Hieda, N. Wada, J. Low Temp. Phys. 150, 032055 (2009).

${ }^{5}$ G. Logvenov, A. Gozar, and I. Bozovic, Science 326, 699 (2009).

6 X.G. Wen and R. Kan, Phys. Rev. B 37, 595 (1988).

7 Y.J. Uemura et al., Phys. Rev. Lett. 62, 2317 (1989); Phys. Rev. Lett. 66, 2665 (1991); Y.J. Uemura, J. Phys.: Condens. Matter 16, S4515 (2004).

8 Y.J. Uemura, Physica B 374-375, 1 (2006).

9 J.K. Chin et al., Nature 443, 961 (2006).

10 M. Greiner, O. Mandel, T. Esslinger, T. W. Hänsch, and I. Bloch, Nature 415, 39 (2002); R. Ramakumar and A.N. Das, Phys. Lett. A 348, 304 (2006).

11 J. Kasprzak et al., Nature 443, 409 (2006).

12 P. Cladé, C. Ryu, A. Ramanathan, K. Helmerson, and W.D. Phillips, Phys. Rev. Lett. 102, 170401 (2009).

13 G. Roati et al., Nature 453, 875 (2008).

14 R. Friedberg and T.D. Lee, Phys. Rev. B 40, 6745 (1989).
15 R. Friedberg, T.D. Lee, and H.-C. Ren, Phys. Rev. B 42, 4122 (1990); Phys. Lett. A 152, 417 and 423 (1991); Phys. Rev. B 45, 10732 (1992).

16 A. Hærdig and F Ravndal, Eur. J. Phys. 14, 171 (1973).

17 I. Trifea and I. Grosu, J. Supercond. Novel Mag 14, 563 (2001).

18 T.M. Hong and J.H. Lin, Phys. Rev. B 52, 7898 (1995).

19 J.M. Ziman, Phil. Mag. 44, 548 (1953).

20 D.F. Goble and L.E. Trainor, Phys. Rev. 157, 167 (1967); D.F. Goble and L.E.H. Trainor, Can. J. Phys. 46, 1867 (1968).

21 R.deL. Kronig and W.G. Penney, Proc. Roy. Soc. (London), A 130, 499 (1930); D.A. McQuarrie, Chem. Educator 1, 1 (1996).

22 R.K. Pathria, Phys. Rev. A 5, 1451 (1972).

23 S. Greenspoon and R.K. Pathria, Phys. Rev. A 8, 2657 (1973); S. Greenspoon and R.K. Pathria, Phys. Rev. A 9, 2103 (1974).

24 H.R. Pajkowski and R.K. Pathria, J. Phys. A: Math. Gen. 10, 561 (1977).

25 R.K. Pathria, Statistical Mechanics, 2nd Ed. (Pergamon, Oxford, 1996).

26 M.F.M. Osborne, Phys. Rev. 76, 396 (1949). 